\title{
Oriented Flip Graphs and Noncrossing Tree Partitions
}

\author{
Alexander Garver焗 and Thomas McConville非 \\ ${ }^{1}$ School of Mathematics, University of Minnesota \\ ${ }^{2}$ School of Mathematics, Massachusetts Institute of Technology
}

\begin{abstract}
Given a tree embedded in a disk, we define two lattices - the oriented flip graph of noncrossing arcs and the lattice of noncrossing tree partitions. When the interior vertices of the tree have degree 3, the oriented flip graph is equivalent to the oriented exchange graph of a type A cluster algebra. Our main result is an isomorphism between the shard intersection order of the oriented flip graph and the lattice of noncrossing tree partitions. As a consequence, we deduce a simple characterization of c-matrices of type A cluster algebras.

Résumé. À partir d'un arbre plongé dans un disque, nous définissions deux réseaux: le "flip graph" orienté des arcs non-croisés et le treillis des partitions non-croisées de l'arbre. Lorsque les sommets intérieurs de l'arbre ont degré 3, le "flip graph" orienté est équivalent au graphe d'échange orienté d'une algèbre amassée de type A. Notre résultat principal est un isomorphisme entre l'ordre d'intersection des tissons du "flip graph" orienté et le treillis des partitions non-croisées de l'arbre. Nous en déduisons une caractérisation simple des c-matrices des algèbres amassées de type A.
\end{abstract}

Keywords. cluster algebra, lattice, noncrossing partitions, Tamari lattice, congruence-uniform, biclosed sets

\section{Introduction}

The facets of a pure, thin simplicial complex form a graph, often called a flip graph, where two facets are adjacent if their intersection is a face of codimension 1. A typical example of a flip graph is the graph of triangulations of a polygon where adjacent triangulations differ by a diagonal flip. This graph is highly connected, a consequence of the fact that it is the 1-skeleton of a polytope known as the associahedron.

Flip graphs often come with a natural acyclic orientation. For example, labeling the vertices of a polygon $1, \ldots, n$ in counter-clockwise order, two diagonals $\{i, k\},\{j, l\}$ cross exactly when $i<j<k<l$. If a triangulation $\mathcal{T}^{\prime}$ is obtained from another triangulation $\mathcal{T}$ by replacing the diagonal $\{i, k\}$ with $\{j, l\}$ where $i<j<k<l$, then we orient the edge $\mathcal{T} \rightarrow \mathcal{T}^{\prime}$. The transitive closure of this relation is the well-studied Tamari order. The lattice structure of the Tamari order and similar posets called Cambrian lattices [11] reveal many interesting combinatorial properties of Catalan objects.

\footnotetext{
†Email: garv0102@umn. edu. Supported by RTG grant NSF/DMS-1148634

‡Email: thomasmc@mit.edu

1365-8050 (c) 2016 Discrete Mathematics and Theoretical Computer Science (DMTCS), Nancy, France
} 
Using the theory of cluster algebras, one may define many different orientations on the graph of triangulations of a polygon. Namely, given a triangulation $\mathcal{T}$, there exists a "natural" acyclic orientation, denoted $\overrightarrow{F G}(\mathcal{T})$, such that $\mathcal{T}$ is the unique source. This construction is given in $\$ 2$

Although these oriented flip graphs have the same underlying graph structure, they tend to be nonisomorphic as posets. The fact that these oriented flip graphs are all lattices comes from a very surprising connection to representation theory: the oriented flip graph is the Hasse diagram of the lattice of torsion classes of some associative algebra. In the full paper, we prove this result combinatorially. More generally, given a polygonal subdivision of a convex polygon, we define a poset of compatible polygonal subdivisions and prove that it forms a lattice.

For our purposes, it is more convenient to define the orientation of the flip graph in terms of the tree dual to a given polygonal subdivision. Namely, given a tree $T$ embedded in a disk, we define in 82 a flip graph $\overrightarrow{F G}(T)$ of maximal collections of pairwise noncrossing geodesics between leaves of $T$.

Theorem 1.1 The oriented flip graph $\overrightarrow{F G}(T)$ is a congruence-uniform lattice.

Congruence-uniformity is a lattice property that will be defined in $\$ 4$. Briefly, a finite congruenceuniform lattice is a lattice that may be constructed by a sequence of interval doublings starting from a 1-element lattice; see Figure 5 . The doubling construction induces a natural edge-labeling on $\overrightarrow{F G}(T)$. This labeling defines a cyclic action $F \mapsto F^{\prime}$ where the up-labels of $F$ are the down-labels of $F^{\prime}$. We refer to this action as Kreweras complementation since it reduces to the usual Kreweras action for the Tamari order.

To prove Theorem 1.1, we define an auxiliary lattice of biclosed sets in $\$ 3$ Then we define a surjective map from biclosed sets to triangulations that identifies $\overrightarrow{F G}(T)$ as a quotient lattice of the lattice of biclosed sets.

Another significant class of Catalan objects are noncrossing partitions [9]. A partition of $[n]:=$ $\{1, \ldots, n\}$ is said to be crossing if there exist two distinct blocks $B, B^{\prime}$ and elements $i, k \in B, j, l \in B^{\prime}$ such that $i<j<k<l$. It is noncrossing otherwise. Noncrossing partitions are well-studied combinatorial objects. They have generalizations to finite Coxeter groups [1] as well as connections to the theory of cluster algebras [12] and the representation theory of finite dimensional algebras [8].

Given a tree $T$ embedded in a disk, we define a noncrossing tree partition to be a partition of the set of interior vertices of $T$ satisfying a similar noncrossing condition; see $\$ 5$. We prove that the poset $\operatorname{NCP}(T)$ of noncrossing tree partitions of $T$ ordered by refinement is a lattice.

As a congruence-uniform lattice, $\overrightarrow{F G}(T)$ admits a alternate ordering known as the shard intersection order $\Psi(\overrightarrow{F G}(T))$, which we recall in $\$ 4$. We prove the following surprising connection between the oriented flip graph and the lattice of noncrossing tree partitions.

Theorem 1.2 There is a Kreweras-equivariant poset isomorphism between $\Psi(\overrightarrow{F G}(T))$ and $\operatorname{NCP}(T)$.

We were motivated to study these posets by a connection to cluster algebras. Given a tree $T$ whose interior vertices have degree 3, one may associate a quiver $Q$ that is mutation-equivalent to a path quiver. The exchange graph on the cluster structure defined by $Q$ comes with a natural acyclic orientation $\overrightarrow{E G}(Q)$. This directed graph is called the oriented exchange graph, and it is isomorphic to $\overrightarrow{F G}(T)$. Translating Theorem 1.2 into the language of cluster algebras, we give a nice characterization of $\mathbf{c}$-matrices associated to $Q$. More details on this connection are given in 86 . 
In this extended abstract, we describe our main results without proof. Proofs will be given in the full version of the paper.

Acknowledgements: We thank the referees for their careful reading and helpful suggestions.

\section{Noncrossing complex}

A tree is a finite connected acyclic graph. Any tree may be embedded in a disk $D^{2}$ such that a vertex is on the boundary if and only if it is a leaf. Unless specified otherwise, we will assume that any tree comes equipped with such an embedding. We will refer to non-leaf vertices of a tree as interior vertices, which we will depict by white dots in our figures. Given trees $T, T^{\prime}$ embedded in $D^{2}$, we consider $T$ and $T^{\prime}$ to be equivalent if there is an isotopy between the spaces $D^{2}-T$ and $D^{2}-T^{\prime}$.

Let $T$ be a tree embedded in $D^{2}$. The embedding of $T$ in $D^{2}$ determines a collection of 2-dimensional regions in $D^{2}$ that we will refer to as faces. A corner of a tree is a pair $(v, F)$ consisting of an interior vertex $v$ and a 2-dimensional face $F$ containing $v$. We denote the set of corners of a tree $T$ by $\operatorname{Cor}(T)$. The embedding that accompanies $T$ endows each interior vertex with a cyclic ordering. Given two corners $(u, F),(u, G) \in \operatorname{Cor}(T)$, we say that $(u, G)$ is immediately clockwise (resp. immediately counterclockwise) from $(u, F)$ about $u$ if $F \cap G \neq \emptyset$ and $G$ is clockwise (resp. counterclockwise) from $F$ according to the cyclic ordering at $u$.

An acyclic path supported by a tree $T$ is a sequence $\left(v_{0}, \ldots, v_{t}\right)$ of vertices of $T$ with $t \geq 1$ such that $v_{i}$ and $v_{j}$ are adjacent if and only if $|i-j|=1$. In particular, vertices of $T$ are not acyclic paths. We typically identify acyclic paths with their underlying vertex sets; that is, we do not distinguish between paths of the form $\left(v_{0}, \ldots, v_{t}\right)$ and $\left(v_{t}, \ldots, v_{0}\right)$.

An arc is an acyclic path whose endpoints are leaves such that for all $i$, the edges $\left(v_{i-1}, v_{i}\right)$ and $\left(v_{i}, v_{i+1}\right)$ are incident to a common face. We say $p$ contains a corner $(v, F)$ if $v=v_{i}$ for some $i=$ $0,1, \ldots, t$ and $F$ is the face that is incident to both $\left(v_{i-1}, v_{i}\right)$ and $\left(v_{i}, v_{i+1}\right)$. Since an arc $p$ divides $D^{2}$ into two components, it determines two disjoint subsets of the set of faces of $T$ that we will call regions. We let $\operatorname{Reg}(p, F)$ denote the region defined by $p$ that contains the face $F$. Observe that if $\operatorname{Reg}(p, F)$ and $\operatorname{Reg}(p, G)$ are the two regions defined by $p$, then $\{\operatorname{Reg}(p, F), \operatorname{Reg}(p, G)\}$ is a set partition of the set of faces of $T$.

A segment is an acyclic path with the same incidence condition as an arc, but whose endpoints are not leaves. Since trees have unique geodesics between any two vertices, if the endpoints of a segment or arc are $v, w$, we may denote the path by $[v, w]$. Let $\operatorname{Seg}(T)$ be the set of segments of $T$.

Definition 2.1 We say that two arcs $p=\left(v_{0}, \ldots, v_{t}\right), q=\left(w_{0}, \ldots, w_{s}\right)$ of a tree $T$ are crossing along a segment $s=\left(u_{0}, \ldots, u_{r}\right)$ if

i) each vertex of $s$ appears in both $p$ and $q$,

ii) if $R_{p}$ and $R_{q}$ are regions defined by $p$ and $q$, respectively, then $R_{p} \not \subset R_{q}$ and $R_{q} \not \subset R_{p}$.

We say they are noncrossing otherwise. The noncrossing complex $\Delta^{N C}(T)$ is the abstract simplicial complex whose simplices are pairwise noncrossing collections of arcs supported by a tree $T$.

Example 2.2 Let $T$ denote the tree shown in Figure 1 and let $p=(7,10,11,12,5)$ and $q=(6,10,11,9,1)$ be arcs of $T$. The arc $p$ contains the corners $\left(10, F_{2}\right),\left(11, F_{5}\right)$, and $\left(12, F_{5}\right)$. The two regions defined by $p$ are $\operatorname{Reg}\left(p, F_{1}\right)=\left\{F_{1}, F_{2}, F_{3}, F_{6}, F_{7}, F_{8}\right\}$ and $\operatorname{Reg}\left(p, F_{4}\right)=\left\{F_{4}, F_{5}\right\}$. The arcs $p$ and $q$ cross along the segment $s=(10,11)$ shown in purple. 


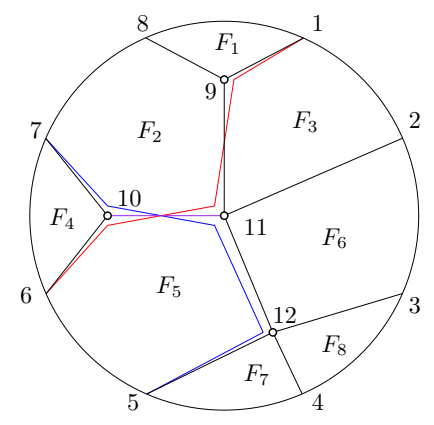

Fig. 1: Two crossing arcs

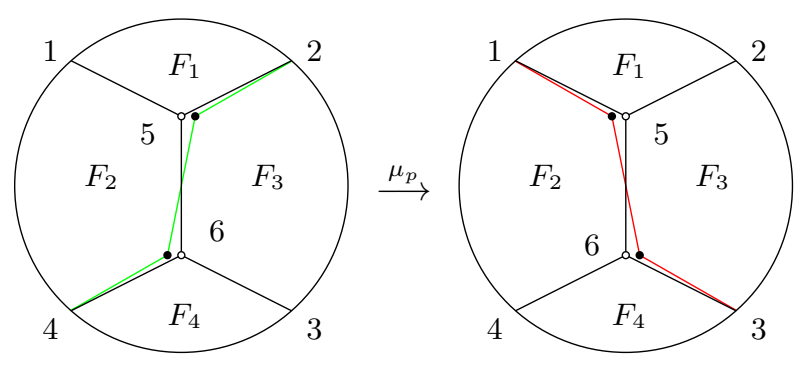

Fig. 2: An oriented flip graph

Lemma 2.3 Let $\mathcal{F}$ be a face of $\Delta^{N C}(T)$ and $(v, F) \in \operatorname{Cor}(T)$ a corner contained in some arc of $\mathcal{F}$. The partially ordered set $\left(\{p \in \mathcal{F}: p\right.$ contains $\left.(v, F)\}, \leq_{(v, F)}\right)$, where $p_{1} \leq_{(v, F)} p_{2}$ if and only if $\operatorname{Reg}\left(p_{1}, F\right) \subset \operatorname{Reg}\left(p_{2}, F\right)$, is linearly ordered. Thus it has a unique maximal element, denoted by $p(v, F)$.

If $p$ is an arc whose vertices all lie on a common face, then $p$ is noncrossing with every arc of $T$. We call such an arc a boundary arc. The reduced noncrossing complex $\widetilde{\Delta}^{N C}(T)$ is the deletion of all boundary arcs from $\Delta^{N C}(T)$. Figure 2 shows the two facets of $\widetilde{\Delta}^{N C}(T)$ where $T$ is the tree drawn in black.

Proposition 2.4 Let $\mathcal{F}$ be a facet of $\Delta^{N C}(T)$ and let $p$ be an arc of $\mathcal{F}$.

i) if $p$ is a nonboundary arc, there are exactly two corners $(u, F),(v, G) \in \operatorname{Cor}(T)$ such that $p=$ $p(u, F)=p(v, G)$. Furthermore, $F$ and $G$ belong to different regions defined by $p$.

ii) if $p$ is a nonboundary arc with $p=p(u, F)=p(v, G)$, there is a unique nonboundary arc $q$ such that $(\mathcal{F} \backslash\{p\}) \cup\{q\}$ is a facet of $\Delta^{N C}(T)$. Furthermore, $q=p\left(u, F^{\prime}\right)=p\left(v, G^{\prime}\right)$ where $\left(u, F^{\prime}\right)$ and $\left(v, G^{\prime}\right)$ are both immediately clockwise or both immediately counterclockwise from $(u, F)$ and $(v, G)$, respectively.

Proposition 2.5 The simplicial complex $\widetilde{\Delta}^{N C}(T)$ is pure (i.e. every facet has the same dimension) and thin (i.e. every codimension 1 simplex is a face of exactly two facets).

Using Proposition 2.4 ii), we refer to the operation sending $\mathcal{F} \longmapsto(\mathcal{F} \backslash\{p\}) \cup\{q\}$ to a new facet of $\widetilde{\Delta}^{N C}(T)$ as a flip of $\mathcal{F}$ at $p$ (see Figure 2 and denote it by $\mu_{p}$. We define the flip graph of $T$, denoted $F G(T)$, to be the graph whose vertices are facets of $\widetilde{\Delta}^{N C}(T)$ and two vertices are connected by an edge if the corresponding facets differ by a single flip. This allows us to define the following fundamental object.

Definition 2.6 Let $\mathcal{F}_{1}, \mathcal{F}_{2} \in F G(T)$ and assume $\mathcal{F}_{2}=\mu_{p} \mathcal{F}_{1}$. Using the notation of Proposition 2.4 ii), we orient the edge connecting $\mathcal{F}_{1}$ and $\mathcal{F}_{2}$ so that $\mathcal{F}_{1} \longrightarrow \mathcal{F}_{2}$ if and only if the corner $\left(u, F^{\prime}\right)$ (resp. $\left.\left(v, G^{\prime}\right)\right)$ is immediately clockwise from the corner $(u, F)$ (resp. $\left.(v, G)\right)$ about vertex $u($ resp. $v)$. The resulting directed graph is the oriented flip graph of $T$, denoted by $\overrightarrow{F G}(T)$.

Example 2.7 Figure 2 and 3 show examples of oriented flip graphs. If every internal vertex of $T$ has degree 3, then $\widetilde{\Delta}^{N C}(T)$ is isomorphic to the dual associahedron (see Figure 3). 

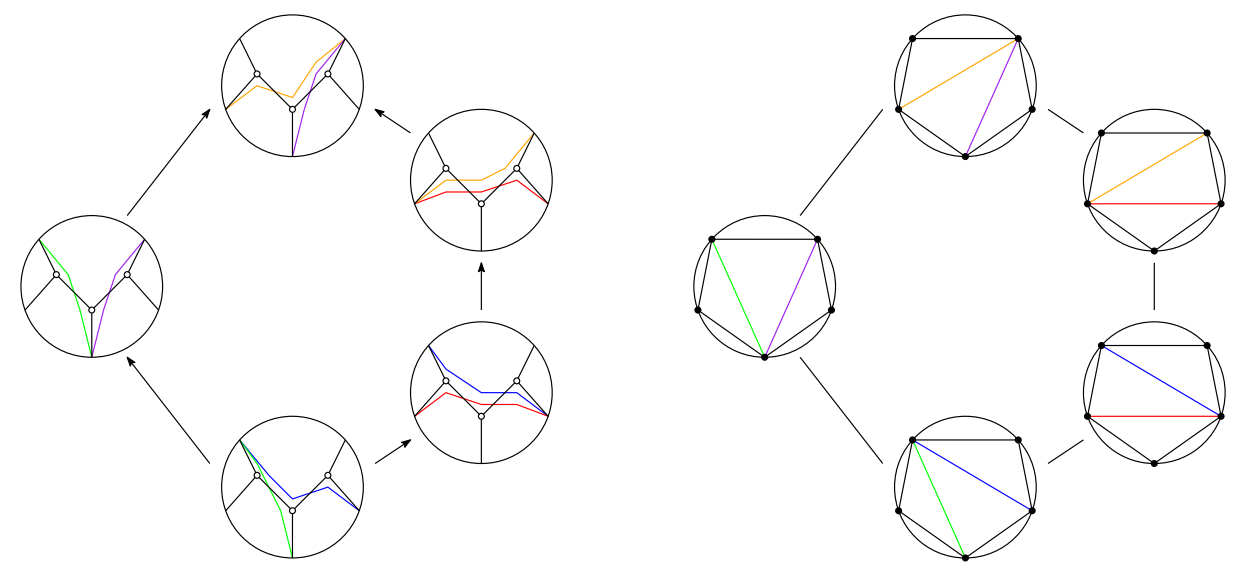

Fig. 3: An oriented flip graph and the corresponding associahedron.

\section{Lattice quotient description of oriented flip graphs}

In this section, we identify the oriented flip graph as both a quotient lattice and a sublattice of another lattice defined below.

Fix a tree $T$ embedded in a disk. Two segments $s, t \in \operatorname{Seg}(T)$ are composable if they are edge disjoint and their union is a segment. We denote the composite segment as $s \circ t$. A subset $X$ of $\operatorname{Seg}(T)$ is closed if for $s, t \in X$, if $s$ and $t$ are composable then $s \circ t \in X$. We say $X$ is biclosed if both $X$ and its complement $\operatorname{Seg}(T)-X$ are closed. We let $\operatorname{Bic}(T)$ denote the poset of biclosed subsets of $\operatorname{Seg}(T)$, ordered by inclusion.

By [7], $\operatorname{Bic}(T)$ is a graded lattice. Moreover, it has the additional structure of a congruence-uniform lattice described in $\$ 4$.

To prove that $\overrightarrow{F G}(T)$ is a lattice, we define a map $\eta$ from $\operatorname{Bic}(T)$ to the facets of $\Delta^{N C}(T)$ and show that it has the structure of a lattice quotient map as in the following lemma.

Lemma 3.1 Let $\eta: L \rightarrow X$ be a surjective map from a finite lattice $L$ to a finite set $X$. If

- $\eta^{-1}(x)$ is a closed interval for all $x \in X$, and

- the maps $\pi_{\downarrow}, \pi^{\uparrow}: L \rightarrow L$ where $\pi_{\downarrow}(y)=\min \eta^{-1}(\eta(y))$ and $\pi^{\uparrow}(y)=\max \eta^{-1}(\eta(y))$ are orderpreserving,

then $X$ inherits the structure of a lattice via $\eta$. Explicitly, for $x_{1}, x_{2} \in X$ and $y_{1}, y_{2} \in L$ with $\eta\left(y_{i}\right)=x_{i}$, $x_{1} \vee x_{2}=\eta\left(y_{1} \vee y_{2}\right)$ and $x_{1} \wedge x_{2}=\eta\left(y_{1} \wedge y_{2}\right)$.

All that remains is to show that the directed graph $\overrightarrow{F G}(T)$ is the Hasse diagram of the lattice structure on the facets of $\Delta^{N C}(T)$ induced by $\eta$.

We define $\eta$ as follows. Let $X \in \operatorname{Bic}(T)$. Given a corner $(v, F)$, let $p_{(v, F)}$ be the (unique) arc supported by $T$ such that for any interior vertex $u$ of $p_{(v, F)}$ distinct from $v$, the following condition holds:

- orienting $p_{(v, F)}$ from $v$ to $u$, the arc $p_{(v, F)}$ turns left at $u$ if and only if $[v, u]$ is in $X$. 

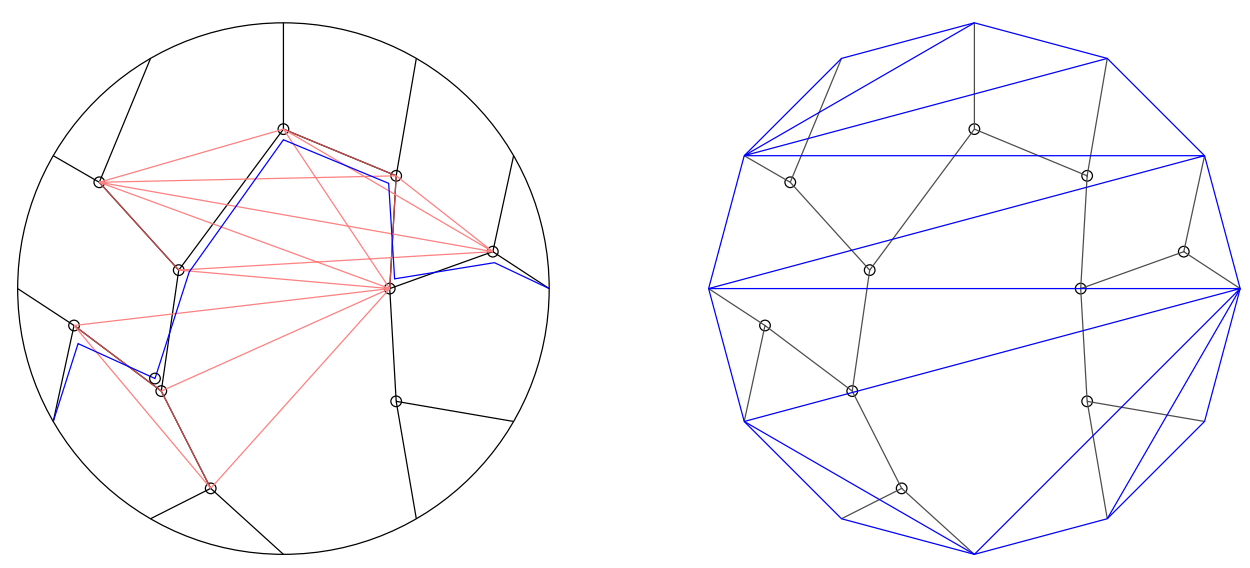

Fig. 4: (left) A blue arc defined by $\eta$ at the circled corner with respect to the red biclosed set of segments; (right) The triangulation defined by $\eta$

One may show that the set of $\operatorname{arcs} p_{(v, F)}$ indexed by the corners of $T$ is a facet of the noncrossing complex. We let $\eta: \operatorname{Bic}(T) \rightarrow \overrightarrow{F G}(T)$ be the map $\eta(X)=\left\{p_{(v, F)}\right\}_{(v, F)}$ where $X \in \operatorname{Bic}(T)$ and arcs $p_{(v, F)}$ are defined as above.

In Figure 4 a biclosed set $X$ of segments is drawn in red. On the left, the $\operatorname{arc} p_{(v, F)}$ is drawn in blue where $(v, F)$ is the circled corner. On the right, the triangulation $\eta(X)$ is drawn in blue.

To obtain a sublattice description, we define a map $\phi: \overrightarrow{F G}(T) \rightarrow \operatorname{Bic}(T)$ as follows. For an arc $p$, let $C_{p}$ be the set of segments $s$ such that $p$ leaves $s$ to the left at each endpoint. Given a triangulation $F$, let $\phi(F)$ be the smallest closed set containing $\bigcup_{p \in F} C_{p}$.

Theorem 3.2 The maps $\eta: \operatorname{Bic}(T) \rightarrow \overrightarrow{F G}(T)$ and $\phi: \overrightarrow{F G}(T) \rightarrow \operatorname{Bic}(T)$ identify $\overrightarrow{F G}(T)$ as a quotient lattice and a sublattice of $\operatorname{Bic}(T)$, respectively.

\section{Congruence-uniformity}

The value of identifying $\overrightarrow{F G}(T)$ as a quotient of $\operatorname{Bic}(T)$ is that $\operatorname{Bic}(T)$ has a much simpler structure. The lattice of biclosed sets of segments has some additional lattice structure that passes to the oriented flip graph via this quotient map. In this section, we consider the congruence-uniform lattice structure of these posets.

Given a subset $I$ of a poset $P$, let $P_{\leq I}=\{x \in P:(\exists y \in I) x \leq y\}$. If $I$ is a closed interval of a poset $P$, the doubling of $P$ at $I$ is the subposet of $P \times \mathbf{2}$, denoted $P[I]$, induced by the set $P_{\leq I} \times\{0\} \sqcup$ $\left(\left(P-P_{\leq I}\right) \cup I\right) \times\{1\}$. Some doublings are shown in Figure 5 . A lattice $L$ is congruence-uniform (or bounded) if it may be constructed from a 1-element lattice by a sequence of doublings.

A CU-labeling is a labeling of the covering relations of a congruence-uniform lattice by the steps in the doubling construction in which they were created. For example, the colors on the edges of Figure 5 form a CU-labeling. More precisely, suppose $\lambda$ is the labeling already constructed for a given congruenceuniform lattice $L$, and let $I$ be a closed interval of $L$. Let $s$ be a label that has not appeared in the labeling 
$\odot$

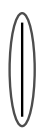

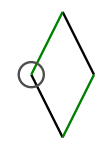

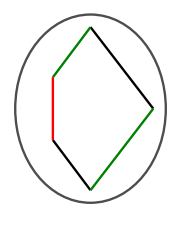

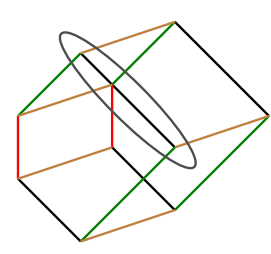
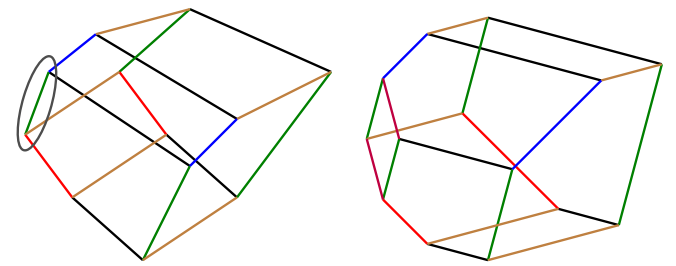

Fig. 5: A sequence of interval doublings

$\lambda$. Then for $(x, \epsilon) \lessdot\left(y, \epsilon^{\prime}\right)$ in $L[I]$, define

$$
\lambda\left((x, \epsilon),\left(y, \epsilon^{\prime}\right)\right)= \begin{cases}s & \text { if } x=y \\ \lambda(x, y) & \text { otherwise. }\end{cases}
$$

Theorem 4.1 [7] Both $\operatorname{Bic}(T)$ and $\overrightarrow{F G}(T)$ are congruence-uniform lattices. Moreover, $\overrightarrow{F G}(T)$ has a $C U$-labeling $\lambda\left(\mathcal{F} \lessdot \mathcal{F}^{\prime}\right)=s$ if $\mathcal{F} \stackrel{s}{\rightarrow} \mathcal{F}^{\prime}$ is an edge of the oriented flip graph.

For an element $x$, let $\lambda_{\downarrow}(x)=\{\lambda(y, x): y \in L, y \lessdot x\}$. Dually, let $\lambda^{\uparrow}(x)=\{\lambda(x, y): y \in L, x \lessdot y\}$.

Lemma 4.2 Let $L$ be a congruence-uniform lattice with $C U$-labeling $\lambda$. For $x \in L$, there exists a unique element $y$ such that $\lambda^{\uparrow}(x)=\lambda_{\downarrow}(y)$.

Lemma 4.2 defines a map $\operatorname{Kr}: L \rightarrow L$, called the Kreweras map, where $\operatorname{Kr}(x)=y$ if $x$ and $y$ are defined as in the lemma. A dual statement to Lemma 4.2 shows that $\mathrm{Kr}$ is a bijection.

Given a congruence-uniform lattice $L$, the shard intersection order can be defined from the labeling $\lambda: \operatorname{Cov}(L) \rightarrow S$ as follows. For $x \in L$, let $y_{1}, \ldots, y_{k}$ be the elements in $L$ such that $\left(y_{i}, x\right) \in \operatorname{Cov}(L)$. Let $\psi(x)$ be the set of labels appearing on covering relations in the interval $\left[\wedge_{i=1}^{k} y_{i}, x\right]$. More precisely,

$$
\psi(x):=\left\{s \in S: \bigwedge_{i=1}^{k} y_{i} \leq w \stackrel{s}{\leftarrow} z \leq x\right\} .
$$

The shard intersection order $\Psi(L)$ is the collection of sets $\psi(x)$ for $x \in L$, ordered by inclusion.

The shard intersection order derives its name from a construction on real hyperplane arrangements due to Nathan Reading. Given an arrangement $\mathcal{A}$ of hyperplanes in $\mathbb{R}^{n}$ with a distinguished chamber, the elements of $\mathcal{A}$ are divided into $(n-1)$-dimensional cones called "shards". The shard intersection order is the lattice of intersections of shards. The connection between the geometric definition and the lattice definition above is explained in the discussion following Theorem 1-7.24 of [13].

\section{Noncrossing tree partitions}

For this section, fix a tree $T$ whose interior vertices all have degree 3 , and let $\widehat{T}$ be the subtree consisting of interior vertices only. Given a subset $B$ of vertices of $\widehat{T}$, there is a unique inclusion-minimal collection $\operatorname{Seg}(B)$ of non-overlapping segments whose endpoints lie in $B$. Each segment $[a, b]$ may be represented by a curve $\gamma$ in the disk with endpoints $a$ and $b$ such that 

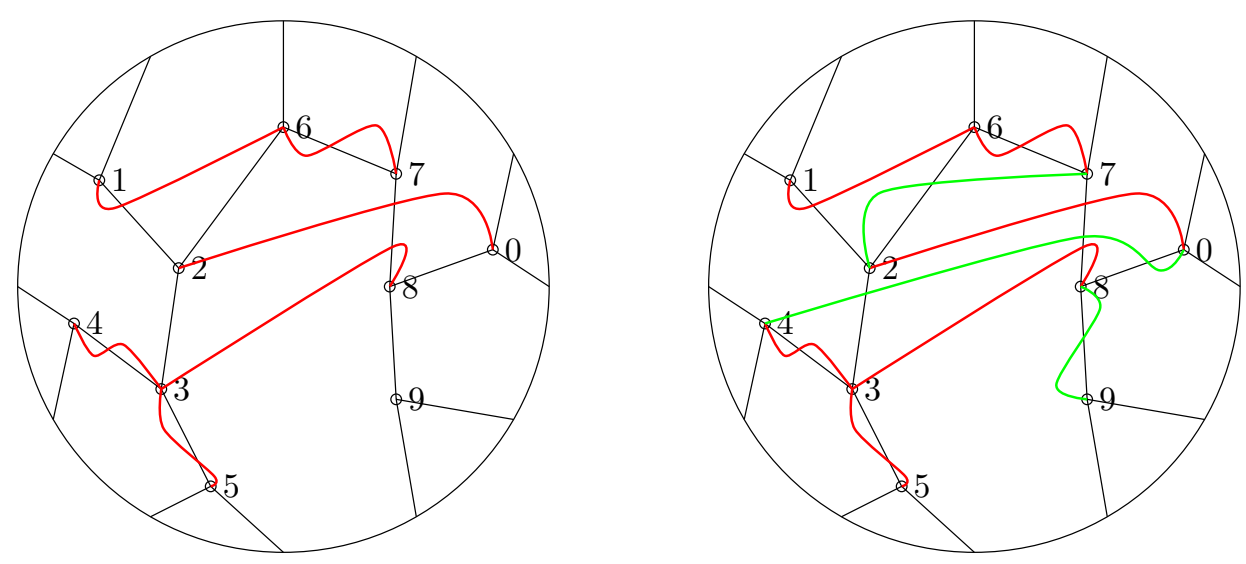

Fig. 6: (left) The noncrossing tree partition $167-20-3458-9$ (right) A noncrossing tree partition in red together with its Kreweras complement $1-27-3-40-5-6-89$ in green

- $\gamma$ leaves each of its endpoints to the right, and

- $\gamma$ does not intersect any edge or vertex of $T$ not in $[a, b]$.

Such a curve $\gamma$ is called a red admissible curve for $[a, b]$. A green admissible curve satisfies the same conditions except that it leaves each of its endpoints to the left. Segments $s$ and $t$ are said to be noncrossing if there exist red admissible curves for $s$ and $t$ that do not intersect. A collection of admissible curves of some noncrossing segments is given in Figure 6.

A noncrossing tree partition of $T$ is a partition $\left\{B_{1}, \ldots, B_{l}\right\}$ of the vertices of $\widehat{T}$ such that for any two distinct blocks $B_{i}, B_{j}$ :

- if $s \in \operatorname{Seg}\left(B_{i}\right), t \in \operatorname{Seg}\left(B_{j}\right)$ then $s$ and $t$ are noncrossing, and

- there do not exist $a \in B_{i}, b, c, d \in B_{j}$ such that $\{a\}=[b, c] \cap[c, d] \cap[b, d]$.

The set $\operatorname{NCP}(T)$ of noncrossing tree partitions of $T$ is partially ordered by refinement. In general, this poset is not isomorphic to the lattice of noncrossing set partitions (for example, see Figure 7).

Proposition 5.1 $\operatorname{NCP}(T)$ is graded lattice. If $T$ has $n$ interior vertices, the number of noncrossing tree partitions of rank $k$ is the Narayana number $\frac{1}{n}\left(\begin{array}{c}n \\ k\end{array}\right)\left(\begin{array}{c}n \\ k+1\end{array}\right)$.

Given a noncrossing tree partition $\pi=\left\{B_{1}, \ldots, B_{l}\right\}$, there is a unique set of segments $S$ such that there exists a collection of red admissible curves for $\operatorname{Seg}(\pi)$ and green admissible curves for $S$ such that no two curves intersect, and the curves form a tree on the interior vertices of $T$. The Kreweras complement $\operatorname{Kr}(\pi)$ is the noncrossing tree partition defined by $S$. Kreweras complementation is a bijection on $\operatorname{NCP}(T)$ such that $\operatorname{rk}(\pi)+\operatorname{rk}(\operatorname{Kr}(\pi))=n-1$.

For $\mathcal{F} \in \overrightarrow{F G}(T)$, let $\psi(\mathcal{F})$ be the most refined set partition on the vertices of $\widehat{T}$ such that any two vertices connected by a segment of $\lambda_{\downarrow}(\mathcal{F})$ are in the same block. For example, if $\mathcal{F}$ is the triangulation in Figure 4 , then $\psi(\mathcal{F})$ is the noncrossing tree partition defined by the red segments in Figure 6 . 


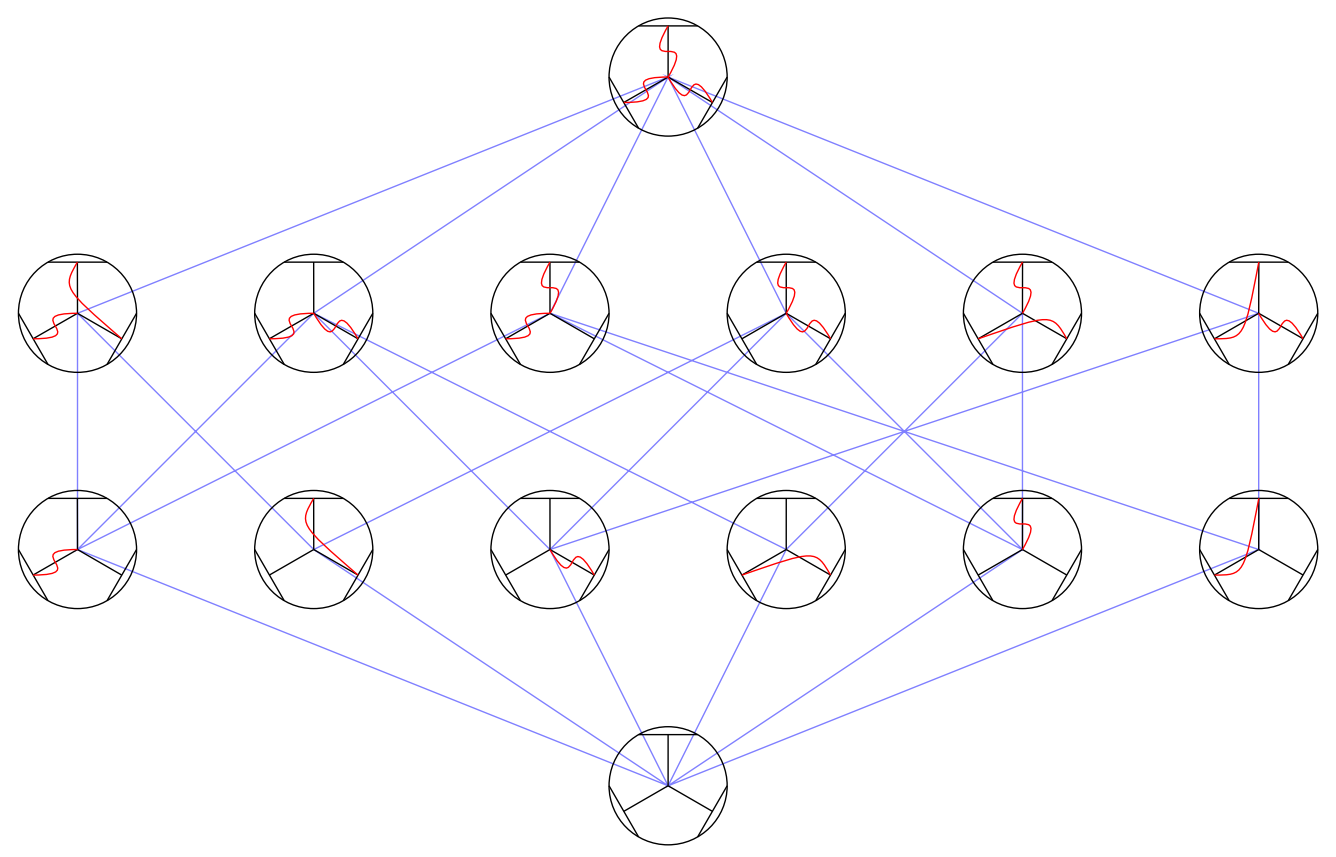

Fig. 7: A noncrossing tree partition lattice

Theorem 5.2 The map $\psi$ is a Kreweras-equivariant bijection from $\overrightarrow{F G}(T)$ to the set of noncrossing tree partitions of $T$. Furthermore, this bijection is a poset isomorphism between the shard intersection order of $\overrightarrow{F G}(T)$ and $\operatorname{NCP}(T)$.

\section{Connection to cluster algebras}

When $T$ is a tree with internal vertices of degree 3, the oriented flip graph of $T$ is isomorphic to the oriented exchange graph [2] of a quiver $Q_{T}$ defined by $T$. By [10], the vertices of the oriented exchange graph of $Q_{T}$ index the clusters in the cluster algebra [5] defined by $Q_{T}$. Also, a quiver defines a family of integer matrices known as c-matrices [6]. Such matrices are related to noncrossing partitions of finite Coxeter groups [12] and many important objects in representation theory [3]. Our main result is that cmatrices of $Q_{T}$ are classified by noncrossing tree partitions of $T$ paired with their Kreweras complement.

A quiver $Q$ is a directed graph without loops or 2-cycles. In other words, $Q$ is a 4-tuple $\left(Q_{0}, Q_{1}, s, t\right)$, where $Q_{0}=[m]:=\{1,2, \ldots, m\}$ is a set of vertices, $Q_{1}$ is a set of arrows, and two functions $s, t$ : $Q_{1} \rightarrow Q_{0}$ defined so that for every $\alpha \in Q_{1}$, we have $s(\alpha) \stackrel{\alpha}{\rightarrow} t(\alpha)$. An ice quiver is a pair $(Q, F)$ with $Q$ a quiver and $F \subset Q_{0}$ frozen vertices with the restriction that any $i, j \in F$ have no arrows of $Q$ connecting them. By convention, we assume $Q_{0} \backslash F=[n]$ and $F=[n+1, m]:=\{n+1, n+2, \ldots, m\}$. Any quiver $Q$ is regarded as an ice quiver by setting $Q=(Q, \emptyset)$.

The mutation of an ice quiver $(Q, F)$ at a nonfrozen vertex $k$, denoted $\mu_{k}$, produces a new ice quiver $\left(\mu_{k} Q, F\right)$ by the three step process:

(1) For every 2-path $i \rightarrow k \rightarrow j$ in $Q$, adjoin a new arrow $i \rightarrow j$. 
(2) Reverse the direction of all arrows incident to $k$ in $Q$.

(3) Delete any 2-cycles created during the first two steps.

We show an example of mutation below with the nonfrozen (resp. frozen) vertices in black (resp. blue).

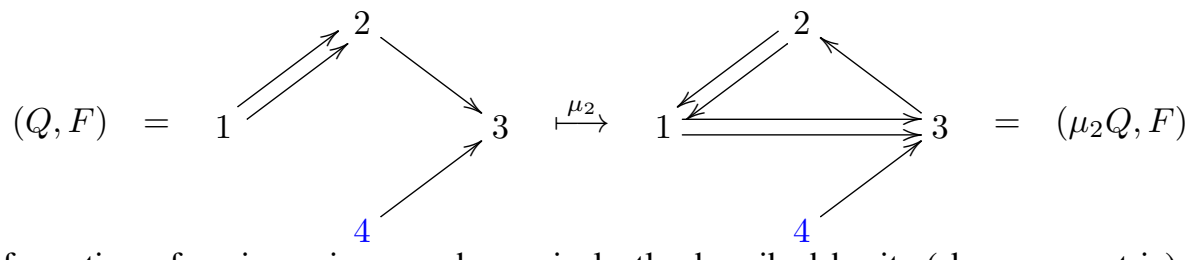

The information of an ice quiver can be equivalently described by its (skew-symmetric) exchange matrix. Given $(Q, F)$, we define $B=B_{(Q, F)}=\left(b_{i j}\right) \in \mathbb{Z}^{n \times m}:=\{n \times m$ integer matrices $\}$ by $b_{i j}:=\#\left\{i \stackrel{\alpha}{\rightarrow} j \in Q_{1}\right\}-\#\left\{j \stackrel{\alpha}{\rightarrow} i \in Q_{1}\right\}$. Furthermore, ice quiver mutation can equivalently be defined as matrix mutation of the corresponding exchange matrix. Given an exchange matrix $B \in \mathbb{Z}^{n \times m}$, the mutation of $B$ at $k \in[n]$, also denoted $\mu_{k}$, produces a new exchange matrix $\mu_{k}(B)=\left(b_{i j}^{\prime}\right)$ with entries

$$
b_{i j}^{\prime}:=\left\{\begin{array}{cl}
-b_{i j} & : \text { if } i=k \text { or } j=k \\
b_{i j}+\frac{\left|b_{i k}\right| b_{k j}+b_{i k}\left|b_{k j}\right|}{2} & : \text { otherwise }
\end{array}\right.
$$

For example, the mutation of the ice quiver above (here $m=4$ and $n=3$ ) translates into the following matrix mutation. Note that mutation of matrices and of ice quivers is an involution (i.e. $\mu_{k} \mu_{k}(B)=B$ ).

$$
B_{(Q, F)}=\left[\begin{array}{ccc|c}
0 & 2 & 0 & 0 \\
-2 & 0 & 1 & 0 \\
0 & -1 & 0 & -1
\end{array}\right] \stackrel{\mu_{2}}{\longmapsto}\left[\begin{array}{rcc|r}
0 & -2 & 2 & 0 \\
2 & 0 & -1 & 0 \\
-2 & 1 & 0 & -1
\end{array}\right]=B_{\left(\mu_{2} Q, F\right)} .
$$

Let $\operatorname{Mut}((Q, F))$ denote the collection of ice quivers obtainable from $(Q, F)$ by finitely many mutations where such ice quivers are considered up to an isomorphism of quivers that fixes the frozen vertices. Such an isomorphism is equivalent to a simultaneous permutation of the rows and first $n$ columns of the corresponding exchange matrices.

Given a quiver $Q$, we define its framed quiver to be the ice quiver $\widehat{Q}$ where $\widehat{Q}_{0}:=Q_{0} \sqcup[n+1,2 n]$, $F=[n+1,2 n]$, and $\widehat{Q}_{1}:=Q_{1} \sqcup\{i \rightarrow n+i: i \in[n]\}$. We define the exchange graph of $\widehat{Q}$, denoted $E G(\widehat{Q})$, to be the (a priori infinite) graph whose vertices are elements of $\operatorname{Mut}(\widehat{Q})$ and two vertices are connected by an edge if the corresponding quivers differ by a single mutation.

The exchange graph of $\widehat{Q}$ has natural acyclic orientation using the notion of $\mathbf{c}$-vectors. We refer to this directed graph as the oriented exchange graph of $Q$, denoted $\overrightarrow{E G}(\widehat{Q})$. Given $\widehat{Q}$, we say that $C=C_{R}$ is a c-matrix of $Q$ if there exists $R \in E G(\widehat{Q})$ such that $C$ is the $n \times n$ submatrix of $B_{R}=\left(b_{i j}\right)_{i \in[n], j \in[2 n]}$ containing its last $n$ columns. That is, $C=\left(b_{i j}\right)_{i \in[n], j \in[n+1,2 n]}$. We let c-mat $(Q):=\left\{C_{R}: R \in\right.$ $E G(\widehat{Q})\}$. A row vector of a c-matrix, $\mathbf{c}_{i}$, is known as a c-vector. Since a $\mathbf{c}$-matrix $C$ is only defined up to a permutations of its rows, $C$ can be regarded simply as a set of $\mathbf{c}$-vectors.

The celebrated theorem of Derksen, Weyman, and Zelevinsky [4, Theorem 1.7], known as the signcoherence of $\mathbf{c}$-vectors, states that for any $R \in E G(\widehat{Q})$ and $i \in[n]$ the $\mathbf{c}$-vector $\mathbf{c}_{i}$ is a nonzero element of $\mathbb{Z}_{\geq 0}^{n}$ or $\mathbb{Z}_{\leq 0}^{n}$. If $\mathbf{c}_{i} \in \mathbb{Z}_{\geq 0}^{n}$ (resp. $\mathbf{c}_{i} \in \mathbb{Z}_{\leq 0}^{n}$ ), we say it is positive (resp. negative). It turns out that for any quiver $Q$, one has that $\mathbf{c}-\operatorname{vec}(Q):=\{\mathbf{c}$-vectors of $Q\}=\mathbf{c}-\operatorname{vec}(Q)^{+} \sqcup-\mathbf{c}-\operatorname{vec}(Q)^{+}$where $\mathbf{c}-\operatorname{vec}(Q)^{+}:=\{$positive $\mathbf{c}$-vectors of $Q\}$. 


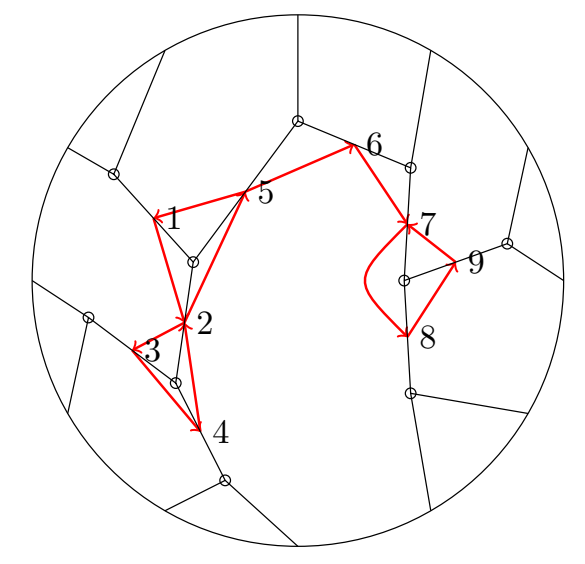

Fig. 8: The quiver $Q_{T}$ associated to a tree $T$.
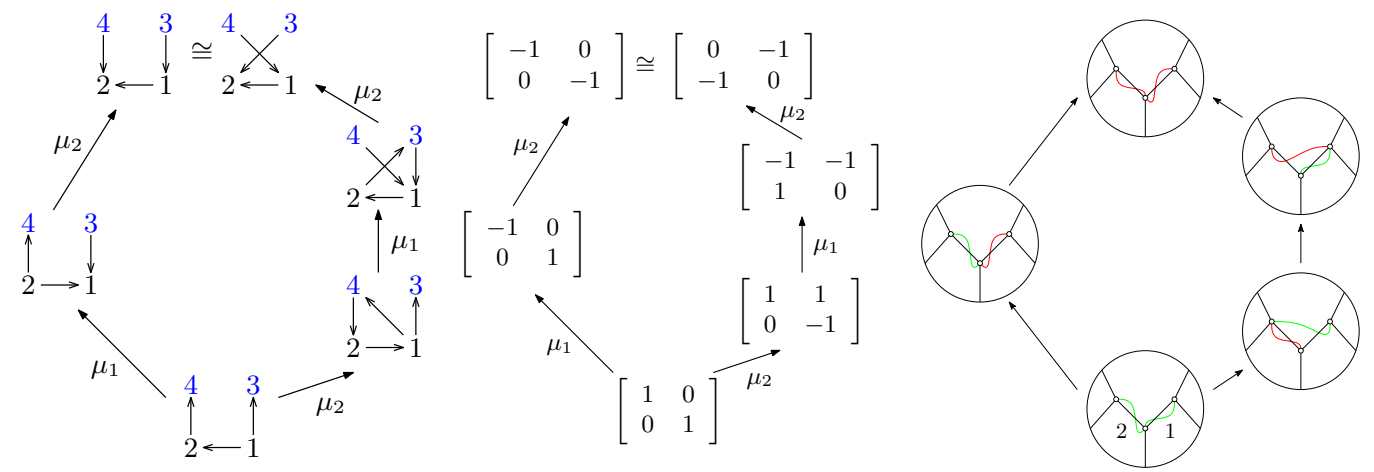

Fig. 9: The oriented exchange graph of of $Q=1 \rightarrow 2$ and the corresponding c-matrices and noncrossing tree partitions with their Kreweras complements.

Definition 6.1 [2] The oriented exchange graph of a quiver $Q$ is the directed graph whose underlying unoriented graph is $E G(\widehat{Q})$ with directed edges $\left(R^{1}, F\right) \longrightarrow\left(\mu_{k} R^{1}, F\right)$ if and only if $\boldsymbol{c}_{k}$ is positive in $C_{R^{1}}$. In Figure 9. we show $\overrightarrow{E G}(\widehat{Q})$ and we also show all of the $\boldsymbol{c}$-matrices inc-mat $(Q)$ where $Q=1 \rightarrow 2$.

Let $T$ be a tree whose internal vertices are of degree 3. Let $Q_{T}$ be quiver whose vertices are in bijection with the edges of $T$ containing no leaves and whose arrows are those of the form $e_{1} \stackrel{\alpha}{\longrightarrow} e_{2}$ satisfying:

i) $e_{1}$ and $e_{2}$ define a corner of $T$,

ii) $e_{2}$ is counterclockwise from $e_{1}$ (see Figure 8).

Theorem 6.2 Assume that $T$ is a tree whose internal vertices are of degree 3 .

1. $\overrightarrow{F G}(T) \cong \overrightarrow{E G}\left(\widehat{Q}_{T}\right)$ as posets and this isomorphism commutes with flips and mutations.

2. The map $\varphi: \operatorname{Seg}(T) \rightarrow \boldsymbol{c}$-vec $(Q)^{+}$defined by $s \mapsto\left(a_{1}, \ldots, a_{n}\right) \in \mathbb{Z}_{\geq 0}^{n}$, where $a_{i}:=1$ if the edge corresponding to vertex $i$ of $Q_{T}$ appears in $s$ and $a_{i}:=0$ otherwise, is a bijection. 
3. The map $\{(\pi, \operatorname{Kr}(\pi))\}_{\pi \in N C P(T)} \rightarrow \boldsymbol{c}$-mat $(Q)$ defined by sending $(\pi, \operatorname{Kr}(\pi))$ to the c-matrix $C$ whose positive c-vectors are $\left\{\varphi(s): s \in \operatorname{Seg}\left(\widetilde{B}_{i}\right)\right.$ where $\left.\widetilde{B}_{i} \in \operatorname{Kr}(\pi)\right\}$ and whose negative $\boldsymbol{c}$-vectors are $\left\{-\varphi(s): s \in \operatorname{Seg}\left(B_{i}\right)\right.$ where $\left.B_{i} \in \pi\right\}$ is a bijection (see Figure 9).

\section{References}

[1] D. Armstrong, C. Stump, and H. Thomas. A uniform bijection between nonnesting and noncrossing partitions. Transactions of the American Mathematical Society, 365(8):4121-4151, 2013.

[2] T. Brüstle, G. Dupont, and M. Pérotin. On maximal green sequences. IMRN, 2014(16):4547-4586, 2014.

[3] T. Brüstle and D. Yang. Ordered exchange graphs. Advances in Representation Theory of Algebras (ICRA Bielefeld 2012), 2013.

[4] H. Derksen, J. Weyman, and A. Zelevinsky. Quivers with potentials and their representations II: applications to cluster algebras. J. Amer. Math. Soc., 23(3):749-790, 2010.

[5] S. Fomin and A. Zelevinsky. Cluster algebras I: foundations. J. Amer. Math. Soc., 15(2):497-529, 2002.

[6] S. Fomin and A. Zelevinsky. Cluster algebras IV: coefficients. Compositio Math., 143(01):112-164, 2007.

[7] A. Garver and T. McConville. Lattice properties of oriented exchange graphs and torsion classes. preprint arXiv:1507.04268, 2015.

[8] C. Ingalls and H. Thomas. Noncrossing partitions and representations of quivers. Compositio Math., 145(06):1533-1562, 2009.

[9] G. Kreweras. Sur les partitions non croisées d'un cycle. Discrete Math., 1(4):333-350, 1972.

[10] T. Nakanishi and A. Zelevinsky. On tropical dualities in cluster algebras. CCM, 565:217-226, 2012.

[11] N. Reading. Cambrian lattices. Advances in Mathematics, 2(205):313-353, 2006.

[12] N. Reading. Clusters, Coxeter-sortable elements and noncrossing partitions. Transactions of the American Mathematical Society, 359(12):5931-5958, 2007.

[13] N. Reading. Lattice theory of the poset of regions. In Lattice Theory: Selected Topics and Applications. Volume 2. Birkhäuser/Springer Basel AG, Basel, forthcoming. 